\title{
ANÁLISIS AL FUTURO DE LAS ARQUITECTURAS DE INTERNET
}

\section{AN ANALYSIS ON THE FUTURE OF THE INTERNET ARCHITECTURES}

\author{
ANALYSE DU FUTUR DES ARCHITECTURES DE L'INTERNET
}

\author{
Shpetimi Latifis \\ Purdue University \\ shplatifis@purdue.edu
}

(Tipo de artículo: REFLEXIÓN. Recibido el 01/03/2011. Aprobado el 25/05/2011)

\begin{abstract}
RESUMEN
Debatir acerca del futuro de los desarrollos más importantes de la ingeniería actual es necesario. Internet es uno de esos desarrollos, por lo tanto necesita discusiones continuas. En este artículo se discute acerca la investigación del futuro de la arquitectura de internet, básicamente en dos corrientes: la de borrón y cuenta nueva, en la que se propone crear una nueva arquitectura, y la de investigación evolutiva, que propone mejorar la arquitectura actual.
\end{abstract}

\section{Palabras clave}

Internet, arquitectura, investigación evolutiva.

\section{Abstract}

It is required to discuss about the future of the most important developments of the current engineering. Internet is one of those developments therefore it needs continuous discussions. This article discusses about the future research on Internet architecture, mainly about two trends: the clean slate, which aims to create a new architecture and evolutionary research, and this of the evolutionary research which aims for the improvement of the current architecture.

\section{Keywords}

Internet, architecture, evolutionary research.

\section{RÉSUMÉ}

C'est nécessaire un débat sur les développements les plus importants du génie actuel. L'Internet est un de ces développements, par conséquent il est nécessaire avoir des discussions continus. Dans cet article on discute sur la recherche de l'architecture de l'Internet, principalement dans deux tendances : ce de table rase, dans laquelle on propose créer une nouvelle architecture, et ce de la recherche évolutive, qui propose améliorer l'architecture actuel.

\section{Mots-clés}

Internet, architecture, recherche. 


\section{INTRODUCCIÓN}

En los últimos años, la comunidad de investigación en redes se ha enfrascado en una conversación acerca de cómo mover el campo -y el propio Internet- hacia adelante. Estos debates tienen lugar en el contexto del enorme éxito de Internet, en el que se ha comenzado a debatir si los investigadores deben centrarse en comprender y mejorar el Internet actual o en diseñar nuevas arquitecturas de red que no estén restringidas por el actual sistema. En última instancia, los investigadores tienen sus propios estilos, a menudo una mezcla única de ambos enfoques. Como aporte a estos debates, en este artículo se presentan los pros y los contras de dos de esos enfoques. Por un lado el de borrón y cuenta nueva, que propone terminar la arquitectura actual y crear una nueva; por otro lado el de la investigación evolutiva, cuya idea es analizar y perfeccionar la arquitectura actual y proyectarla para un mejor futuro.

\section{BORRÓN Y CUENTA NUEVA}

El Internet tiene un éxito innegable -un experimento de investigación que se escapó del laboratorio para convertirse en una parte importante de la infraestructura mundial de comunicaciones. Las semillas del éxito de Internet residen en su "insuficientemente" diseño -una red minimalista proporciona un simple servicio de mejor esfuerzo para la entrega de paquetes acoplado con computadores programables en los puntos finales. Al principio, esas decisiones de diseño eran muy importantes debido a que reducen las barreras a la innovación en nuevas aplicaciones -creadas por cualquier persona que quiera programar estos computadores-, y tecnologías de enlace -que se pueden adaptar fácilmente si son compatibles con el modelo básico de paquetes de entrega [1]. Esto ha llevado la innovación más allá de lo que se hubiera imaginado.

Teniendo en cuenta ese éxito de Internet y su aparente flexibilidad para la innovación, el enfoque de borrón y cuenta nueva en la investigación puede parecer extraño, incluso superfluo. Sin embargo, nada podría estar más lejos de la verdad. De hecho, este enfoque es importante para que el campo de la creación de redes pueda madurar y convertirse en una verdadera disciplina, y tener una futura arquitectura de Internet que sea confiable para la sociedad. Además, es posible que no esté totalmente en desacuerdo con la investigación evolutiva. Los resultados de estos procesos investigativos pueden $-\mathrm{y}$ deben- ayudar a guiar la evolución de la arquitectura actual de Internet, y un rediseño de este tipo puede ser necesario para que Internet evolucione de forma continua hacia una infraestructura segura, confiable y rentable. Lo más importante, como comunidad de investigación, es que debe plantar las semillas que permitan futuros experimentos de investigación para "salir del laboratorio" [2].

\subsection{Hacia una disciplina de red}

El éxito de Internet no significa que el campo de las redes esté maduro, ni mucho menos. El Internet ha crecido y cambiado mucho más rápido que la propia comprensión de cómo diseñar, construir y operar grandes redes. Este es un fenómeno común en la ingeniería. Las grandes catedrales medievales se construyeron mucho antes de que el campo de la ingeniería civil estuviera maduro. Como resultado, muchas de esas catedrales colapsaron por su propio peso después de décadas de construidas. Incluso las catedrales colapsadas fueron una valiosa experiencia de aprendizaje a lo largo del camino hacia un enfoque más riguroso para el diseño y construcción de grandes estructuras. Ellas fueron un paso en el camino, no el destino. La forma en que se diseñan hoy los grandes edificios refleja más que mejoras incrementales en las técnicas de ingeniería, está principalmente más enfocada al problema.

Cada vez que el Internet enfrenta nuevos retos, desde los temores al colapso de congestión de finales de 1980 hasta las acuciantes preocupaciones de seguridad cibernética de hoy, se introducen nuevos parches para hacerle frente - al menos parcialmente- a los problemas. Sin embargo, todavía no se tiene nada que se acerque a una disciplina para crear, analizar y operar los protocolos de red, por no hablar de las combinaciones de protocolos y mecanismos vistos en las redes reales. Las redes todavía no es una verdadera disciplina académica, basada en modelos rigurosos y en técnicas probadas para orientar a los diseñadores y operadores. Verificar cualquier clase de red o libro de texto, con todas las descripciones de protocolos existentes en lugar de un tratamiento arriba abajo de las "leyes" o "reglas de oro" que rigen el diseño, el análisis, y el funcionamiento de estos protocolos. Dada la importancia crítica de las redes de comunicación, se necesita que este campo madure y se convierta en una disciplina que se puede aplicar con seguridad en la práctica, y que se pueda enseñar con eficacia a los estudiantes [3].

Si bien el estudio actual de Internet es claramente una parte importante de maduración para el campo, no es suficiente; también se necesita exploración que no tiene restricciones para los artefactos actuales. Para ser claros, ignorar los artefactos de hoy no significa ignorar la realidad. Cualquier nuevo diseño debe lidiar con las limitaciones prácticas -como la velocidad de la luz o las limitaciones en computación, memoria y recursos de ancho de banda- y los requisitos de diseño -para objetivos como eficiencia, seguridad, privacidad, fiabilidad, rendimiento, facilidad de gestión, y otros. Sin embargo, un proceso de diseño de borrón y cuenta nueva puede permanecer libre de las cuantiosas minucias de los protocolos de hoy y las prácticas operativas, y los desafíos de implementación incremental.

Un proceso de diseño borrón y cuenta nueva puede derribar los supuestos básicos de la arquitectura actual, como hacerlo cuando es posible lograr escalabilidad sin depender del direccionamiento jerárquico, el tráfico de ruta directamente en el nombre de un servicio más que la dirección de una máquina, o 
tener nociones de identidad que no pueden ser falsas. Esta exploración de borrón y cuenta nueva puede conducir a valorar nuevos diseños que llenen los grandes espacios, ampliando nuestro conocimiento y experiencia. Esta exploración puede, de forma tal vez más importante, dar lugar a nuevas metodologías para diseñar redes y protocolos; y cómo implementar estas nuevas ideas en la Internet actual, aunque sin duda un tema meritorio en sí mismo, a veces debe ser secundario metas más amplias de profundizar nuestra comprensión de este campo. La medida del éxito de la investigación debe ser la mayor profundidad de nuestra comprensión, no sólo la amplitud del despliegue.

Sin embargo, la investigación en borrón y cuenta nueva en redes no puede quedar en diseños de lápiz y papel. Además de nuevas ideas, y rigurosos modelos teóricos y de análisis, se necesita impulsar más nuestras ideas en aplicaciones reales e, idealmente, en implementaciones. Los momentos "Eureka" que conducen a un progreso real suceden cuando encontramos sorpresas, cuando sucede algo que nunca podría haberse planificado o previsto. Construir, evaluar e implementar sistemas reales, expone las ideas nacientes a la cruda luz del día, y nos da la retroalimentación necesaria para ayudar a nuestras ideas a crecen más nítidas y fuertes a medida que direccionamos los contratiempos inesperados $y$ limitaciones, y aceptar las limitaciones prácticas y los requisitos de diseño que sin saberlo estábamos ignorando.

Construir e implementar nuestros diseños es algo más que el último paso en la evaluación de una idea, forma parte de un ciclo continuo de la investigación, refinando constantemente el problema, los modelos, y las soluciones hasta que surja una comprensión más completa. Este enfoque para la investigación de redes debe sonar familiar, ya que es exactamente como se diseñó y construyó en sus comienzos ARPAnet, llevando a los impresionantes avances que se han visto desde hace 40 años, cuando el primer mensaje fue entregado por la red que hoy se ha dado en llamar "el Internet". En ese momento, la idea de que la ARPAnet finalmente superara a las redes de telecomunicaciones establecidas en su época era inconcebible para la mayoría de las personas [4]. Sin embargo, hoy se sabe cómo resultó la historia.

\subsection{Hacia un internet digna de confianza}

La Internet está mostrando signos de vejez. Problemas de seguridad generalizados como correo electrónico no deseado, ataques de denegación de servicio, suplantación de identidad, entre otros, son sólo los síntomas más visibles. Además, no maneja bien hosts móviles si los usuarios están movimiento o migran máquinas virtuales de un computador a otro. El modelo de servicio con mejor esfuerzo de Internet es un pobre encuentro para muchas aplicaciones en tiempo real, como IPTV y servicio de videoconferencia. Internet no es lo suficientemente confiable, debido a fallas en los equipos, errores de software, y los errores de configuración. Administrar una gran red es demasiado costoso, a menudo cuesta más que el equipo de base y es tremendamente propenso a errores. Internet consume demasiada energía, en una época de grave preocupación por el calentamiento global; no parece dispuesto a manejar los ataques provenientes de un sinnúmero de dispositivos sensores pequeños que tienen el potencial de revolucionar nuestro mundo; y la lista sigue y sigue.

Muchos de estos apremiantes desafíos están profundamente arraigados en las decisiones de diseño subyacentes a Internet, y no pueden ser solucionables sin cambios arquitectónicos fundamentales. Por ejemplo, muchos problemas de seguridad se refieren a las nociones de identidad débiles de Internet, y particularmente a la facilidad de falsificación de casi todo, desde las direcciones IP, los nombres de dominio hasta las direcciones de correo electrónico para enrutar información. En la Arquitectura actual no se instalan fácilmente las fuertes nociones de identidad necesarias. La movilidad es difícil de manejar porque las direcciones IP son jerárquicas y estrechamente unidas con la escalabilidad de los protocolos de enrutamiento. Romper este acoplamiento puede requerir una nueva relación entre la denominación, el direccionamiento y el enrutamiento. La gestión de la red es difícil debido a la actual "división del trabajo" entre los protocolos distribuidos ejecutados sobre los elementos de la red y los sistemas de gestión que sólo indirectamente pueden ajustar los botones de muchos de estos protocolos expuestos. Resolver estos problemas puede requerir que se revisen algunos de los principios más básicos subyacentes a la Internet de hoy.

La investigación de borrón y cuenta nueva permite explorar los diseños radicalmente nuevos, para ver si son alternativas viables para la solución que tenemos ahora. Algunas de estas soluciones pueden muy bien tener una ruta incremental para implementarse. Pero, como dijo la leyenda del beisbol americano Yogi Berra: "Tienes que ser muy cuidadoso si no sabes a dónde vas, porque puede ser que no llegues allí"; y esta investigación puede ser la ayuda que requerida para determinar dónde se debe ir. El diseño de borrón y cuenta nueva también puede ayudar a que se decida qué partes de Internet no deberían cambiar. Tal vez, a pesar de los desafíos que enfrenta la actual Internet, fundamentalmente no se haga mucho para mejor algunas dimensiones, por ejemplo en seguridad, sin tener que pagar un precio demasiado alto a lo largo de otra dimensión. Esta investigación puede ayudar para lograr entender las ventajas y desventajas, y para guiar las decisiones acerca de qué cambiar [5].

Por último, un masivo cambio sea a la vez necesario y posible. A pesar de que permite la innovación en las aplicaciones y las tecnologías de enlace, la arquitectura de Internet en sí es muy resistente al cambio. En su rediseño, se podrá prestar la atención necesaria a este problema. Hacer más programable el interior de la red y permitir diseños independientes 
múltiples para convivir en paralelo, es un comienzo prometedor en este sentido. Tal vez el Internet del futuro podría tener las semillas para su propia y constante reinvención desde el interior. Ya se observan los primeros frutos de este tipo de pensamiento de borrón y cuenta nueva, en la definición de software para infraestructuras de redes como Open-Flow -http://www.openflowswitch.org/-, que está siendo desplegando en varias empresas, centros de datos y redes troncales. Incluso en infraestructuras experimentales como GENI y Federica, diseñadas para permitir múltiples experimentos simultáneos con nuevas arquitecturas de redes, se ven ejemplos de este tipo de cambios.

Los cambios fundamentales de este tipo es son efecto posibles y ya está empezando a ocurrir, debido a los esfuerzos de la investigación de borrón y cuenta nueva a largo de los años últimos. Además, puede ser el cambio más sustancial que puede suceder en los próximos años. Dado que la Internet suplanta gran parte del circuito de conmutación de las redes telefónicas, ¿es descabellado pensar que algo más podría suplantar a la Internet, o alterarla significativamente al punto que no sea posible reconocerla desde las descripciones en los libros de texto sobre redes de hoy?

\section{INVESTIGACIÓN EVOLUTIVA}

Primero vamos a identificar la diferencia principal entre los dos enfoques. La investigación evolutiva tiene como objetivo entender el comportamiento del Internet actual, identificar los problemas existentes 0 emergentes, y resolverlos de acuerdo con dos grandes limitaciones: la compatibilidad retrasada -inter-operar sin problemas con la arquitectura heredada de Internet-, y la implementación incremental - se debe utilizar un nuevo protocolo o tecnología como principio, incluso si no está implementado a nivel mundial. Por otro lado, la investigación de borrón y cuenta nueva tiene como objetivo diseñar una nueva arquitectura para el "Internet del futuro", que sea significativamente mejor -en términos de rendimiento, seguridad, resistencia y otras propiedades- que la que utiliza el Internet actual y sin verse limitada por su misma arquitectura.

\subsection{Impacto en el mundo real de la investigación de borrón y cuenta nueva}

La investigación de borrón y cuenta nueva no es algo nuevo. De hecho, hay una larga historia de esfuerzos y es posible aprender algo analizando si los protocolos y arquitecturas anteriores de borrón y cuenta nueva se han adoptado o no. Por citar algunos ejemplos, considere redes activas, calidad del servicio en ingeniería de tráfico de redes y control de admisión, la conectividad del protocolo de red CLNP, los protocolos de transporte como XCP, o las arquitecturas de enrutamiento entre dominios como Nimrod. También existe un gran número de protocolos que son más o menos compatibles con versiones anteriores pero no realmente de despliegue incremental, tales como IPv6, IP de reparto múltiple entre dominios, RSVP, IntServ,
IPsec, o S-BGP. Posiblemente, estos protocolos no han visto el despliegue a gran escala, por lo menos hasta ahora. En cambio, el "mundo real" ha adoptado enfoques evolutivos como NATs, almacenamiento en caché y redes de distribución de contenido, DiffServ, aplicaciones adaptativas, y diversos mecanismos de seguridad -como seguridad de anfitrión final, sistemas de detección de intrusiones, y filtros de enrutamientoque funcionan bien con la arquitectura heredada. ¿Por qué la investigación en la arquitectónica de borrón y cuenta nueva, o incluso los protocolos y los diseños que intentan ser compatible con versiones anteriores, a menudo no se adoptan en la práctica?

En la economía industrial es bien conocido que una tecnología emergente, que está sujeta a las externalidades de la red, probablemente no será capaz de reemplazar a una tecnología ampliamente implementada aunque inferior mientras haya costos involucrados en el proceso [6]. En cambio, la pregunta más pertinente es si la nueva tecnología ofrece un servicio nuevo y valioso que la tecnología actual no puede proporcionar directa o indirectamente. En otras palabras, ¿puede el valor adicional de una nueva tecnología, en relación con la tecnología establecida, compararse con el costo de transición?

No es suficiente para la arquitectura de borrón y cuenta nueva ser "mejor" que la arquitectura actual de Internet. Primero debe tener un impacto que sea capaz de sustituirla, de lo contrario continuará como un mero ejercicio intelectual. La cuestión del impacto en el mundo real es lo que diferencia los enfoque de investigación y el diseño de borrón y cuenta nueva y la evolutiva. Y por lo menos hasta ahora, los defensores de la investigación de borrón y cuenta nueva no han demostrado casos en los que sus nuevas aplicaciones o servicios no puedan ser, directa o indirectamente, construidos para la Internet actual. Es cierto que la promesa de una "futura Internet" segura y digna de confianza es atractiva, pero no convincente: no hay forma de proporcionar garantías de seguridad con un modelo de amenazas abiertas. Además, es muy probable que una nueva arquitectura de interconexión tenga más errores de diseño y de implementación, y más huecos de seguridad que la arquitectura de la actual Internet -que han sido "depurados" por más de 30 años.

Los proponentes del diseño de borrón y cuenta nueva enfatizan que no se quedarán con "diseños de papel", ya que van a construir y experimentar las arquitecturas propuestas en bancos de pruebas, tales como GENI. Pero, ¿qué probaría eso? Hace 10 ó 20 años también se aplicaron y probaron varios protocolos previos de borrón y cuenta nueva; el problema no fue la falta de aplicación o de experimentación, el hecho fue que esos protocolos no podían competir con las tecnologías existentes, teniendo en cuenta los beneficios reales que ofrecían a los usuarios y los costos involucrados en la transición tecnológica. GENI o los bancos de pruebas no pueden ayudar en los estudios fundamentalmente por cuestiones 
económicas. Además, estos bancos de pruebas no son utilizados por aplicaciones y personas reales, y no operan bajo las limitaciones económicas y políticas del mundo real. Los principios de ARPANET tuvieron éxito porque no era sólo un banco de pruebas: se utilizó también como una red de producción, conectando algunas universidades y laboratorios de investigación, mientras que al mismo tiempo los investigadores de redes podían experimentar con nuevos protocolos y tecnologías.

Otra creencia popular es que la arquitectura del Internet actual es el resultado de borrón y cuenta nueva pensando antes en los años 1960 y 1970. Sin embargo, no se debe ignorar que la conmutación de paquetes o TCP/IP no fue una invención que "salió de la nada", es el resultado de un proceso evolutivo que se inició a partir de la multiplexación síncrona en las redes de conmutación de circuitos, pasando a la multiplexación asíncrona y luego al reenvío de datagramas. Además, la arquitectura ARPANET fue sólo una de varias arquitecturas en competencia, como SNA de IBM, DECnet, X.25 de la UIT, Xerox pup, SITA HLN, o CYCLADES, y fue a través de un largo proceso evolutivo que la primera finalmente prevaleció.

\subsection{Está realmente osificada la arquitectura de Internet}

Uno de los argumentos principales para la investigación de borrón y cuenta nueva ha sido que la arquitectura actual de Internet está osificada, especialmente en las capas centrales de apilado de protocolos -IP y TCP-, y que los ISPs no tienen incentivos para adoptar algunas innovaciones arquitectónicas. Esta es una visión bastante negativa de lo que sucede. La arquitectura de Internet mapea una diversidad cada vez mayor de tecnologías de capa de enlace para una serie cada vez mayor de aplicaciones y servicios. Para soportar esta innovación en las capas de menor a mayor de la arquitectura, los protocolos centrales de la arquitectura deben evolucionar muy lentamente, de modo que formen una base estable desde la que la diversidad y la complejidad pueda surgir.

Para utilizar una analogía biológica, algunos desarrollo Redes Reguladoras de Genes se establecieron en el Cámbrico -hace unos 510 millones de años- y no han evolucionado significativamente desde entonces. Estos GRNs se conocen como núcleos evolutivos, y hoy se sabe que son en gran parte responsable de los aspectos principales de todos los planes corporales de los animales. Por ejemplo, el corazón de una mosca de la fruta y el corazón de un ser humano, a pesar de tener morfologías distintas, desarrollaron el uso de la misma base cardiaca GRN. Los núcleos evolutivos representan una base estable que puede envolver la diversidad y la complejidad de los procesos de más alto nivel [7]

3.3 Una agenda para investigar la Internet Evolutiva En lugar de pensar en Internet como un artefacto diseñado en el pasado y que ahora debe ser rediseñado, lo que se debe es empezar a pensar en Internet como un ecosistema en evolución que se ve afectado por diversas disciplinas, y cómo se estudia. Su evolución es controlada, no sólo por la tecnología, sino también por la economía global, las ideas creativas de millones de personas, y un conjunto de cambio constante de "las presiones del medio ambiente" y las restricciones. Nuestra misión, entonces, como investigadores de Internet, es primero medir y comprender el estado actual de este ecosistema, predecir hacia dónde se dirige y los desafíos a los que pronto se enfrentará, y crear lo que podría denominarse como mutaciones inteligentes: las innovaciones que pueden, en primer lugar, evitar o resolver esos desafíos, y en segundo lugar, las innovaciones que pueden ser adoptadas por la actual arquitectura de forma que sea compatible hacia atrás y que se despliegue incrementalmente. Se trata de una agenda de investigación pragmática que puede tener un impacto real en millones de personas.

En lugar de bancos de pruebas, la investigación evolutiva necesita diversos recursos experimentales que se integrarán en la Internet actual. En primer lugar, se necesita una densa infraestructura de "monitores de Internet " de diversos tipos, que permitirán medir con precisión lo que está sucediendo actualmente en este ecosistema en constante evolución. Es desconcertante que -a pesar del enorme valor del proyecto Route Views- todavía no tenga una forma precisa para medir la topología entre dominios de Internet. Además, no tiene una estimación de cuántos flujos de tráfico hay entre dos sistemas autónomos, aunque el tráfico entre dominios de la matriz determina en gran medida la economía de la Internet global. Además, no es posible conocer cómo utiliza la población a la Internet y a la Web a través del tiempo y el espacio. Dado que esta brecha en el conocimiento se incrementa, es preocupante que pronto no podrá seguir nuestra propia creación, y mucho más para influir en su futuro.

La investigación en Internet evolutiva se beneficiaría enormemente con una amplia infraestructura de monitoreo si fuese posible que operáramos nuestros propios ISP experimentales. Esto sería una verdadera red TCP/IP, ejecutando todos los protocolos de la arquitectura actual de Internet, presente en muchos puntos de intercambio, igualándolos abiertamente con otros ISP y proveedores de contenido, y transportando de forma real el tráfico que pertenece a los usuarios de Internet. Una forma de hacerlo podría ser que las universidades utilicen este ISP experimental para realizar parte de su tráfico de forma gratuita, comprendiendo que se trata de una red de investigación y por lo tanto el tráfico podría sufrir "mutaciones" experimentales de la arquitectura de Internet. Esto es diferente al Internet2 o NLR, que son redes de producción, y sin duda muy diferente a bancos de prueba como GENI.

\subsection{Después de todo, ¿dónde está la ciencia?}

Los proponentes del diseño de borrón y cuenta nueva argumentan que su enfoque conduce a una ciencia del 
diseño de la red, que a veces también es referido como "ciencia de las redes", pero que es confuso porque el término se emplea también en otras disciplinas para referirse al estudio de sistemas complejos con gráficos dinámicos modelos y técnicas de análisis de redes. También afirman que la investigación evolutiva no es una ciencia, sino una colección de "hacks" y de mejoras incrementales. Esta es una posición engañosa. Algunos avances en la investigación de redes han sido resultados de la investigación evolutiva. Por ejemplo, los principales resultados en el control de congestión y la gestión activa de colas han resultado de los intentos por comprender y mejorar el TCP, el descubrimiento de las propiedades fundamentales del tráfico de Internet y la topología, el diseño de protocolos de comunicación innovadoras peer-to-peer, o el desarrollo de redes de inferencia end-to-end, así como los métodos de tomografía de la red [8].

Un dominio del conocimiento no se convierte en ciencia porque por el hecho de estar basado en marcos de optimización limpios o porque prueben resultados de profundidad sobre modelos simples. La buena ciencia requiere relevancia para el mundo real, medidas y validación experimental, hipótesis comprobables, y modelos con capacidad predictiva.

\section{CONCLUSIONES}

Quienes trabajan en los procesos investigativos de borrón y cuenta nueva argumentan que el diseño de redes es un campo del conocimiento todavía joven. Que el éxito de Internet es algo que se debe admirar y celebrar, pero no debemos estar conformes con comprensión actual de este campo, o ver la arquitectura de Internet como una figura en piedra. Tal vez una nueva generación de investigadores y profesionales podrán convertir el futuro Internet en algo vagamente parecido a su predecesor. Quizás esta futura red podrá acomodarse al cambio de manera más amplia y profunda que como lo hace la Internet de hoy. La voluntad de dar un paso atrás y diseñar desde cero, es una parte importante del repertorio de investigación que pueda permitir estos avances en el campo, y en la propia Internet.
Quienes se orientan por la investigación evolutiva se preguntan acerca del porqué varios respetados investigadores de Internet decidieron trabajar con el enfoque de borrón y cuenta nueva. No puede ser sólo el asunto de la financiación, de eso están seguros. Ellos encuentran alegría y orgullo en la idea de poder rediseñar internet desde cero, en que pueden evitar todos los errores anteriores y perfeccionarlos a este momento. Si no queremos que suene como diálogo de ciencia-ficción, es importante seguir fomentando la evolución del Internet actual, teniendo un impacto positivo en la forma como millones de personas viven, trabajan y se comunican.

\section{REFERENCIAS}

[1] W. B. Arthur. "Competing Technologies and Lock-In by Historical Small Events: The Dynamics of Allocation Under Increasing Returns". Discussion Paper 43. Center for Econopmics Policy Research. Stanford Univesity. 1985.

[2] Chakravarty S. "Experimental evidence on product adoption in the presence of network externalities". Review of Industrial Organization, Vol. 23, No. 3-4, pp. 233-254. 2003.

[3] D. Fudenberg \& J. Tirole. "Pricing a network good to deter entry". Journal of Industrial Economics, Vol. 58, No. 4, pp. 373-390. 2000.

[4] E. R. Berndt, Z. Griliches \& N. J. Rappaport. "Econometric estimates of price indexes for personal computers in the 90s", Journal of Econometrics, Vol. 68, No. 3, pp. 241-257. 1995.

[5] J. M. Gallaugher \& Y. Wang. "Understanding network effect in software markets: evidence from web server pricing", MIS Quarterly, Vol. 26, No. 4, pp. 303-327. 2002.

[6] B. W. Arthur. "Competing technologies, increasing returns, and lock-in by historical events". The Economic Journal, Vol. 99, No. 394, pp. 116-131. 1989.

[7] C. Dovrolis \& T. Streelman. "Evolvable network architectures: What can we learn from biology?" ACM SIGCOMM Computer Communications Review, Vol. 40, No. 2. 2010.

[8] C. L. Webb. "Brussels Sprouts a Microsoft Ruling", http://www.washingtonpost.com/wp-dyn/articles/ A597102004Mar15.html. [Sept. 2010]. 